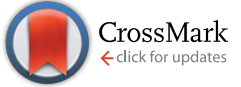

Cite this: RSC Adv., 2017, 7, 3293
Received 22nd October 2016 Accepted 15th December 2016

DOI: 10.1039/c6ra25651a

www.rsc.org/advances

\section{Hetero-assembly of a $\mathrm{Li}_{4} \mathrm{Ti}_{5} \mathrm{O}_{12}$ nanosheet and multi-walled carbon nanotube nanocomposite for high-performance lithium and sodium ion batteries $\dagger$}

\begin{abstract}
Y. Tian, Z. L. Wu, G. B. Xu, L. W. Yang* and J. X. Zhong
A nanocomposite composed of crystalline $\mathrm{Li}_{4} \mathrm{Ti}_{5} \mathrm{O}_{12}$ (LTO) nanosheets and multi-walled carbon nanotubes (denoted as LTO/MWCNTs) is prepared for lithium/sodium storage via a facile hetero-assembly process driven by the screening effect of electrostatic repulsion and following thermal treatment. The morphology and microstructure characterizations reveal that LTO nanosheets are embedded in a highly porous and electrically conductive MWCNT network, thereby producing robust inner-connected architectures. Owing to improved electron and ion transportation during electrochemical reaction rendered by a unique integrated architecture of two-dimensional LTO nanosheets and MWCNTs, the nanocomposite exhibits excellent lithium and sodium storage performances. For lithium ion batteries, the anode delivers reversible capacity of $149.7 \mathrm{~mA} \mathrm{~h} \mathrm{~g}^{-1}$ at $10 \mathrm{C}$ after 500 cycles. As anode for sodium ion batteries, it has reversible capacity of $82 \mathrm{~mA} \mathrm{~h} \mathrm{~g}^{-1}$ at $5 \mathrm{C}$. The prepared LTO/MWCNT nanocomposite has great potential as a high-performance anode material for dual storage applications in lithium and sodium ion batteries.
\end{abstract}

\section{Introduction}

In past decades, ever-increasing demands in automobile and stationary energy storage applications, such as electric vehicles (EVs) and renewable energy integration, has spurred fast development of rechargeable, long-life, high-capacity and lowcost energy storage devices. Li-ion batteries (LIBs) have been a favorable power source unit in portable electronics and EVs because of their advantages of high capacity, long cycle time, and environmental benignity. Unfortunately, there are some disadvantages for LIBs and the most important one is the exhaustion of lithium in the near future. In this background, room temperature sodium-ion batteries (SIBs) have been re-highlighted for next-generation rechargeable batteries, benefiting from abundant and inexpensive sodium resources. Nevertheless, many restrictions yet exist for these battery technologies, such as separators, electrolytes and electrode materials. Particularly, high-performance electrode material is a crucial factor for both LIBs and SIBs. Despite great efforts devoted to develop suitable new electrode materials such as $\mathrm{Si}{ }^{1}$ transition-metal oxides, ${ }^{2,3}$ transition-metal dichalcogenides ${ }^{\mathbf{4 , 5}}$ and NASICON-type compounds, ${ }^{6-8}$ electrode materials

Hunan Key Laboratory of Micro-Nano Energy Materials and Devices, School of Physics and Optoelectronics, Xiangtan University, Hunan 411105, China. E-mail: ylwxtu@xtu. edu.cn

$\dagger$ Electronic supplementary information (ESI) available. See DOI: 10.1039/c6ra25651a simultaneously with high-rate capability and ultrastability still remain a challenge. Additionally, only a few reports pay attention to the dual storage properties with both lithium and sodium of electrode materials.

Spinel $\mathrm{Li}_{4} \mathrm{Ti}_{5} \mathrm{O}_{12}$ (LTO) has been regarded as a promising electrode material for LIBs owing to its flat operating plateau at a high potential of $\sim 1.55 \mathrm{~V} v s$. $\mathrm{Li} / \mathrm{Li}^{+}$, zero-strain feature towards lithium insertion/extraction, and excellent environmental benignity. ${ }^{9-15}$ Recently, this well-known 'zero-strain' material has been also exploited as anode with a work potential of $\sim 0.91 \mathrm{~V}$ vs. $\mathrm{Na} / \mathrm{Na}^{+}$for SIBs. ${ }^{16-20}$ The relatively higher storage voltages for LIBs/SIBs make LTO intrinsically much safer than carbon anode. While LTO-related materials are being extensively studied for LIBs, their application in SIB has been explored relatively less. It is reported recently that LTO nanorods and C-LTO nanowires have been synthesized and demonstrated good $\mathrm{Na}$ storage performance. ${ }^{21,22}$ However, practical applications of LTO in high-performance LIBs and SIBs are hampered because of the unsatisfactory rate capability due to kinetic issues associated with poor electrical conductivity ( $c a$. $10^{-13} \mathrm{~S} \mathrm{~cm}^{-1}$ ) and small ion diffusion coefficient ( $\mathrm{ca} \cdot 10^{-9}$ to $10^{-13} \mathrm{~cm}^{2} \mathrm{~s}^{-1}$ for $\mathrm{Li}^{+}$and $10^{-16} \mathrm{~cm}^{2} \mathrm{~s}^{-1}$ for $\mathrm{Na}^{+}$). To overcome these hurdles, one effective strategy is to increase the conductivity of nano/microstructured LTO including nanoparticles, ${ }^{23,24}$ nanowires, ${ }^{12,22}$ nanosheets, ${ }^{25,26}$ hollow microspheres ${ }^{27-29}$ by adding conductive materials, such as carbon nanotubes (CNTs), graphene or conducting polymers to form nanocomposites. 
Obviously, the route to formation of these LTO-based nanocomposites is much critical to obtain superior electrochemical performance. Several novel approaches were reported within recent years to prepare nanocomposites by directly growing LTO on carbonaceous materials. Shen et al. reported the in situ growth of $\mathrm{Li}_{4} \mathrm{Ti}_{5} \mathrm{O}_{12}$ on multi-walled carbon nanotubes for high rate lithium ion batteries. ${ }^{30} \mathrm{Du}$ et al. has successfully synthesized $\mathrm{Li}_{4} \mathrm{Ti}_{5} \mathrm{O}_{12}$ with a conductive network of carbon via in situ spray pyrolysis. ${ }^{31}$ These techniques largely boost homogeneousness of nanocomposites as forming connected networks. Nevertheless, besides the usually rigorous synthetic conditions, it is also difficult to manipulate the mass ratio of LTO to carbonaceous materials and then to optimize the performance of LIBs/SIBs further. Although physical mixing of nano/ microstructured LTO with carbonaceous materials is a simple and conventional method to form nanocomposites, the lack of uniformity in the nanocomposites is always a serious drawback due to their different density. Consequently, it is desirable to develop a simple but effective approach to homogeneously integrate nano/microstructured LTO with CNTs, graphene or conducting polymers, thereby spurring the application of LTObased nanocomposites in high-performance LIBs and SIBs.

Among various nanoarchitectures, two-dimensional (2D) nanosheets hold great promise in high-performance batteries because of the short paths enabling rapid ion diffusion, large exposed surface area accommodating more $\mathrm{Li} / \mathrm{Na}$, as well as abundant ion insertion channels. ${ }^{32-35}$ Previously, we reported a facile method to synthesize highly-crystalline LTO nanosheets via the topotactic transformation of trivalent lanthanide (for example, $\mathrm{Gd}^{3+}$ ) doped ultrathin layered $\mathrm{Li}_{1.81} \mathrm{H}_{0.19} \mathrm{Ti}_{2} \mathrm{O}_{5} \cdot x \mathrm{H}_{2} \mathrm{O}$ (H-LTO) at $700{ }^{\circ} \mathrm{C}^{36,37}$ Herein, based on previous works, we prepare a nanocomposite composed of crystalline LTO nanosheets and multi-walled carbon nanotubes (denoted as LTO/ MWCNTs) via a facile hetero-assembly process and followed thermal treatment. Owing to unique inner-connected architecture of 2D LTO nanosheets embedded in highly porous and electrically conductive MWCNTs networks, the developed nanocomposite exhibits considerable lithium and sodium storage performances. For LIBs, the anode delivers reversible capacity of $149.7 \mathrm{~mA} \mathrm{~h} \mathrm{~g}^{-1}$ at $10 \mathrm{C}$ after 500 cycles. As anode for SIBs, it has reversible capacity of $82 \mathrm{~mA} \mathrm{~h} \mathrm{~g}^{-1}$ at $5 \mathrm{C}$. The results provide an available method to prepare LTO-based nanocomposites for practical applications in high-performance LIBs and SIBs.

\section{Experimental details}

\subsection{Synthesis of highly-crystalline LTO nanosheets}

Crystalline LTO nanosheets were prepared by a modified hydrothermal method followed by calcination. ${ }^{32,36,37}$ In the typical synthesis procedure, $1.7 \mathrm{ml}(5 \mathrm{mM})$ of tetrabutyltitanate, $0.03 \mathrm{~g}$ of $\mathrm{GdCl}_{3} \cdot 6 \mathrm{H}_{2} \mathrm{O}$, and $0.189 \mathrm{~g}$ of $\mathrm{LiOH} \cdot \mathrm{H}_{2} \mathrm{O}$ were thoroughly mixed in $20 \mathrm{ml}$ of ethanol at room temperature. The solution was mixed completely with a magnetic stirrer in a closed container for $24 \mathrm{~h}$ followed by $25 \mathrm{ml}$ of deionized water being added to the container. After stirring for $0.5 \mathrm{~h}$, the solution was transferred into a $50 \mathrm{ml}$ Teflon-lined stainless autoclave and placed in an oven at $180^{\circ} \mathrm{C}$ for $36 \mathrm{~h}$. The white powder on bottom of the reactor was collected, washed with ethanol 3 times, and dried at $80^{\circ} \mathrm{C}$ for $6 \mathrm{~h}$. Finally, the white powder was heated at $700{ }^{\circ} \mathrm{C}$ for $6 \mathrm{~h}$ in a horizontal tube furnace in air to obtain crystalline LTO NSs.

\subsection{Preparation of LTO nanosheets/MWCNTs nanocomposite}

Firstly, MWCNTs were oxidized by nitric acid through a simple way. ${ }^{38}$ Secondly, based on the process described in our previous work, ${ }^{39}$ Crystalline LTO nanosheets were dispersed in $100 \mathrm{ml}$ ionic solution containing $0.2 \mathrm{M} \mathrm{NH}_{4} \mathrm{HCO}_{3}(1.58 \mathrm{~g}$ ) to form Solution A, and oxidized MWCNTs were dispersed in $100 \mathrm{ml}$ of deionized water to produce Solution B. Solution A was added dropwise to Solution B under stirring. As completely mixed, black fluffy precipitates sink to the bottom within ten minutes. The precipitates were collected via vacuum filtration, and further heat-treated at $200{ }^{\circ} \mathrm{C}$ for $6 \mathrm{~h}$ to obtain LTO/ MWCNTs nanocomposite. For comparison, a reference sample (denoted as W-LTO/MWCNTs) was also prepared by vacuum filtration of LTO nanosheets and MWCNTs colloid in deionized water and following thermal treatment at $200{ }^{\circ} \mathrm{C}$ for $6 \mathrm{~h}$.

\subsection{Materials characterization}

The crystal structures of the synthesized samples were determined by powder X-ray diffraction (XRD) using a copper $K_{\alpha}$ radiation source $(\lambda=0.154 \mathrm{~nm})$ at $40 \mathrm{kV}$ and $200 \mathrm{~mA}$ in steps of 0.02. Data were recorded ranging from $10^{\circ}$ to $90^{\circ}$. The morphology and microstructure of the synthesized samples were characterized using scanning electron microscopy (SEM, JEOL, JSM-6490) and transmission electron microscopy (TEM, JEOL 2100) equipped with selected area electron diffraction (SAED) and an Oxford energy dispersive X-ray spectroscopy (EDS) system. Raman spectra were recorded on a Renishaw inVia system with a laser operating at $\lambda=532 \mathrm{~nm}$ as excitation source. Fourier transform infrared (FTIR) spectra measurements were carried out on a FTS-3000 Fourier transform infrared spectrophotometer.

\subsection{Electrochemical characterization}

The electrochemical tests were measured using two-electrode CR2032 type coin cells. Working electrodes for lithium and sodium cells were prepared by pasting a mixture of the active materials, carbon black and polyvinylidene fluoride (PVDF) at a weight ratio of $80: 10: 10$ onto $\mathrm{Cu}$ foil which acted as a current collector. The electrodes were dried at $80{ }^{\circ} \mathrm{C}$ for $6 \mathrm{~h}$ in air, and then at $120^{\circ} \mathrm{C}$ in vacuum for another $12 \mathrm{~h}$ and pressed. The mass of every electrode was weighed accurately using an electronic balance. The mass of the active materials was controlled in the range of 1-2 mg. Coin cells were assembled in an argon-filled gloved box. For the lithium cells, the metallic lithium foil was used to the counter electrode, $1 \mathrm{M} \mathrm{LiPF}_{6}$ in ethylene carbonate (EC)/dimethyl carbonate (DEC) (1:1, volume ratio) as electrolyte, and Celgard 2400 polypropylene as separator, whereas sodium cells were assembled with sodium as the counter electrode, the glass fiber as separator (Whatman 
GF/D), and $1 \mathrm{M} \mathrm{NaClO}_{4}$ in ethylene carbonate (EC)/propylene carbonate (PC) (1:1, volume ratio) as electrolyte. The charge/ discharge measurements were performed at various current densities over a voltage range of 1 to $2.5 \mathrm{~V}\left(v s\right.$. $\left.\mathrm{Li}^{-} \mathrm{Li}^{+}\right)$and 0.5 to $2.5 \mathrm{~V}$ (vs. $\mathrm{Na} / \mathrm{Na}^{+}$) using a multi-channel battery test system (NEWARE BTS-610). Cyclic voltammetry (CV) measurements were carried out on an electrochemical workstation (CHI660D) in the voltage range of 1 to $2.5 \mathrm{~V}\left(v s\right.$. $\left.\mathrm{Li} / \mathrm{Li}^{+}\right)$and 0.5 to $2.5 \mathrm{~V}$ ( $v s$. $\mathrm{Na} / \mathrm{Na}^{+}$) at a scan rate of $0.1 \mathrm{mV} \mathrm{s}^{-1}$. Electrochemical impedance spectroscopy (EIS) measurement was carried out in the frequency range from $0.01 \mathrm{~Hz}$ to $100 \mathrm{kHz}$ on a CHI660D electrochemical workstation. All tests were carried out at room temperature.

\section{Results and discussions}

Fig. 1 shows a brief illustration of hetero-assembly process to prepare the LTO/MWCNTs nanocomposite. Previous studies revealed that stable MWCNTs colloid is often produced in water due to strong electrostatic repulsion of adjacent MWCNTs (see Fig. 1) after the MWCNTs oxidized by nitric acid are negatively charged in water (molecular solution) due to the ionization of carboxylic groups and hydroxyl groups. It suggests that positively-charged particles tend to be attracted to neutralize negatively-charged MWCNTs to screen the electrostatic repulsion among MWCNTs and disturb stability accompanying coprecipitation. Inspired by a deliberation in our previous study, ${ }^{39}$ in the synthesis of the LTO/MWCNTs nanocomposite, crystalline LTO nanosheets are dispersed in the solution containing $0.2 \mathrm{M} \mathrm{NH}_{4} \mathrm{HCO}_{3}$ with high ionic strength to make them absorbed plenty of positive and negative charged ions. As the LTO nanosheets in $\mathrm{NH}_{4} \mathrm{HCO}_{3}$ solution are added into colloidal MWCNTs solution, the LTO nanosheets promptly assemble with MWCNTs due to screening effect of electrostatic repulsion among MWCNTs. As a result, hetero-assembly of LTO nanosheets and MWCNTs is readily produced and spontaneously precipitates in a macroscopically visible process. As shown in Fig. 1, after $6 \mathrm{~h}$, black precipitate falls to the bottom leaving the solution colorless, indicating efficient embedding of additive LTO nanosheets in MWCNTs networks. The uniform precipitate is stable without any separation of LTO nanosheets even after several days, implying that the MWCNTs have a well-defined and interconnected 3D network and the embedded LTO nanosheets attach tightly to MWCNTs. Finally, with thermal treatment of the collected precipitate at $200{ }^{\circ} \mathrm{C}$, residual $\mathrm{NH}_{4} \mathrm{HCO}_{3}$ decomposes completely and the LTO/MWCNTs nanocomposite was obtained. The resultant LTO/MWCNTs nanocomposite demonstrates improved electrical conductivity compared with the LTO (see Fig. S1 $\dagger$ ). For comparison, when the LTO nanosheets are dispersed in pure water, the above hetero-assembly phenomenon is not observed. In this case, the LTO nanosheets precipitate out by themselves instead due to relatively high density, while the MWCNTs still keep well-dispersed (see the upper of Fig. 1) because the electrostatic repulsion among negatively charged MWCNTs is not screened.

The LTO/MWCNTs nanocomposite is characterized by the combination techniques of XRD, FTIR spectra and Raman scattering. Fig. 2a shows the XRD patterns of the prepared nanocomposite with a weight ratio of $4: 1$ for LTO nanosheets and MWCNTs. All peaks are well indexed to those of spinel LTO nanosheets (JCPDS card no. 49-0207, S. G.: $F d 3 m$ ) and oxidized MWCNTs. The sharp and intense peaks from LTO nanosheets suggest high crystallinity. The peak at $26.4^{\circ}$ ascribed to the (002) peak of MWCNTs is rather weak, indicating that the stacking of MWCNTs is suppressed in the LTO/MWCNTs nanocomposite, and consequently leading to the weakening of the reflection peak. This result also suggests that LTO nanosheets are highly dispersed into MWCNTs networks and integrate with the latter tightly via co-assembly driven by screening effect of electrostatic repulsion among MWCNTs in the solution with high ionic strength. Fig. 2b shows the FTIR spectra of as-obtained LTO nanosheets, MWCNTs and LTO/MWCNTs nanocomposites. In FTIR spectra, the following functional groups are identified in oxidized MWCNTs: the hydroxyl groups $\left(\mathrm{H}-\mathrm{OH}, 3435 \mathrm{~cm}^{-1}\right.$, $\mathrm{C}-\mathrm{OH}, 1023 \mathrm{~cm}^{-1}$ ), the carbonyl groups (COOH, $1690 \mathrm{~cm}^{-1}$ ), and $\mathrm{C}=\mathrm{C}$ stretching vibration $\left(1565 \mathrm{~cm}^{-1}\right)$, which is in agreement with the literature. ${ }^{38}$ The FTIR spectra of the nanocomposites with different weight ratios of LTO nanosheets and MWCNTs exhibit the same peak positions, indicating that there are no chemical reactions between LTO nanosheets and oxidized MWCNTs. Fig. 2c displays the Raman spectra of asprepared LTO/MWCNTs nanocomposites to confirm the chemical composition. The Raman bands located in the range from 200 to $1000 \mathrm{~cm}^{-1}$ show typical $\mathrm{Li}_{4 / 3} \mathrm{Me}_{5 / 3} \mathrm{O}_{4}$ spinel features

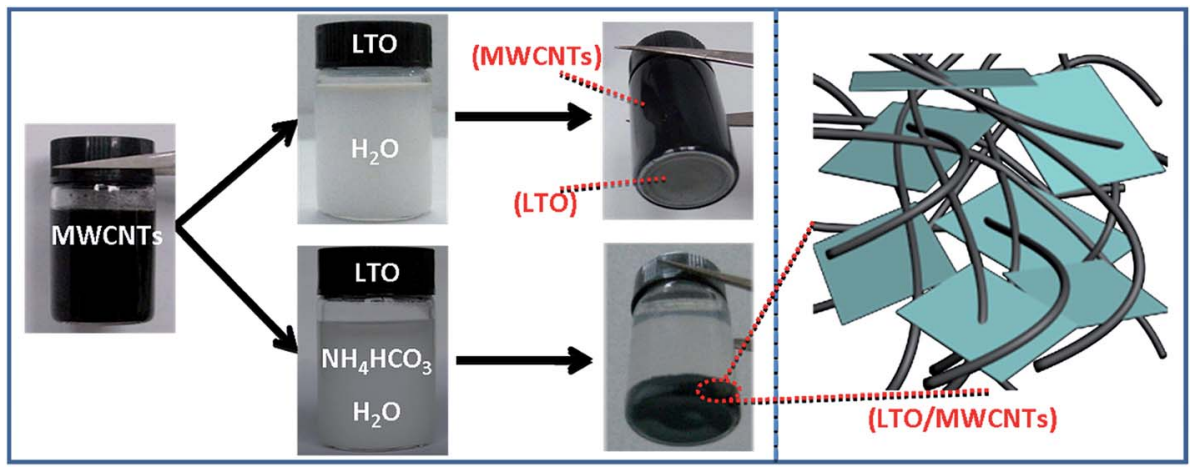

Fig. 1 Photograph illustration and schematic formation process for the LTO/MWCNTs nanocomposite. 

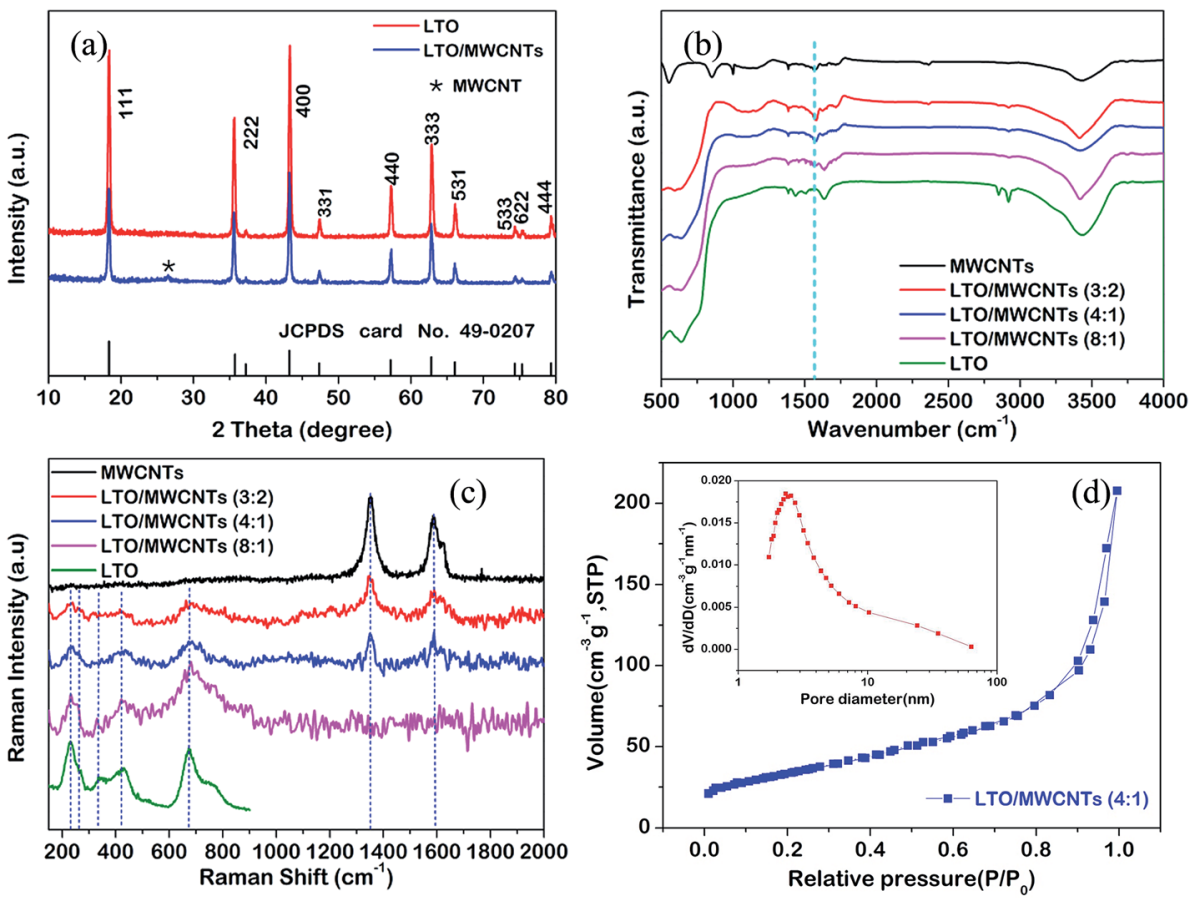

Fig. 2 (a) XRD patterns of LTO nanosheets and LTO/MWCNTs nanocomposite with a weight ratio of 4 : 1; (b) FTIR spectra of LTO nanosheets, MWCNTs and LTO/MWCNTs nanocomposites with different weight ratios; (c) corresponding Raman spectra of the samples; (d) the BET surface area and pore size distribution of LTO/MWCNTs (4:1) nanocomposites.

with all five active Raman phonon modes $\left(\mathrm{A}_{1 \mathrm{~g}}+\mathrm{E}_{\mathrm{g}}+3 \mathrm{~F}_{2 \mathrm{~g}}\right)$ indicative of cubic spinel LTO and in agreement with the peaks of pure LTO NSs in our previous report. ${ }^{40}$ The strong band of $\mathrm{A}_{1 \mathrm{~g}}$ at $678 \mathrm{~cm}^{-1}$ with a shoulder at $754 \mathrm{~cm}^{-1}$ is assigned to the stretching vibrational mode of Ti-O covalent bonding in $\mathrm{TiO}_{6}$ octahedra. The stretching vibrational mode of $\mathrm{Li}-\mathrm{O}$ ionic bonds located in the $\mathrm{LiO}_{4}$ tetrahedra $\left(\mathrm{E}_{\mathrm{g}}\right)$ is present at $431 \mathrm{~cm}^{-1}$. Three bands $\left(\mathrm{F}_{2 \mathrm{~g}}\right)$ at $340 \mathrm{~cm}^{-1}, 268 \mathrm{~cm}^{-1}$ and $234 \mathrm{~cm}^{-1}$ originate from the vibration of lithium, which is octahedrally-coordinated by oxygen. Besides these Raman modes of spinel LTO, two strong peaks, denoted as the disordered peak $(\mathrm{D}$, centered at $\sim 1350$ $\mathrm{cm}^{-1}$ ) and the graphitic peak (G, at $\sim 1580 \mathrm{~cm}^{-1}$ ) are also observed from the LTO/MWCNTs nanocomposites. The D peak is ascribed to edge planes and disordered structures associated with the disruption of the symmetrical hexagonal graphitic lattice, and the $\mathrm{G}$ peak corresponds to the first-order scattering of the in-plane stretching mode for symmetric $\mathrm{sp}^{2}$ carbon, which is consistent with those from MWCNTs. There are no observable Raman peaks between 1200 and $2000 \mathrm{~cm}^{-1}$ from LTO NSs for all Raman characterizations. The Raman results further confirm the co-existence of MWCNTs and LTO nanosheets in the LTO/MWCNTs nanocomposites. In addition, the BET analysis for LTO/MWCNTs nanocomposites (Fig. 2d) manifests a surface area of $120.8 \mathrm{~m}^{2} \mathrm{~g}^{-1}$ and pore size distribution ranging from 2 to $100 \mathrm{~nm}$.

The morphology and microstructure are determined by SEM and TEM. Fig. 3a depicts a typical SEM image, demonstrating sheet-like morphology of the as-obtained LTO nanosheets. Fig. 3b shows a typical TEM image of as-obtained LTO nanosheets. As shown in Fig. 3c, the crystalline region with clear lattice fringes has an inter-planar spacing of approximately $0.48 \mathrm{~nm}$, corresponding to the (111) atomic planes of spinel LTO, confirming high crystallinity. Fig. 3d presents the assembled LTO/MWCNTs nanocomposite.

Further TEM observation (see Fig. 3e and f) reveals that LTO nanosheets are embedded in MWCNTs matrix and MWCNTs attach to LTO nanosheets tightly, thereby producing robust inner-connected architectures. In such assembled LTO/ MWCNTs nanocomposite, the MWCNTs act as faster transmission channel network to connect the LTO nanosheets, which is certainly beneficial to electrical and ionic transfer during the electrochemical reaction of LTO, thereby improving energy storage performance in LIBs and SIBs.

The Li-ion storage performances of all samples were systematically evaluated by cyclic voltammetry (CV) and galvanostatic charge-discharge cycling in half cells. Fig. 4a shows the CV curves of the nanocomposite with a weight ratio of $4: 1$ for LTO nanosheets and MWCNTs (LTO/MWCNTs $(4: 1)$ ) at a scanning rate of $0.1 \mathrm{mV} \mathrm{s}^{-1}$ in the potential range from 1.0 to $2.5 \mathrm{~V}\left(v s . \mathrm{Li}^{+} / \mathrm{Li}\right)$. Both of them have well-defined redox peaks, which are attributed to the redox reaction of $\mathrm{Ti}^{4+} / \mathrm{Ti}^{3+}$, and in good agreement with lithium insertion and extraction in the spinel LTO lattice. ${ }^{9}$ The redox peaks of the LTO/MWCNTs nanocomposite are expectably stronger than that of LTO nanosheets owing to improving electron transport and lithium ion diffusion with addition of MWCNTs. The voltage difference between the anodic and cathodic peaks $(\Delta E)$ reflects the degree of polarization. The $\Delta E$ of the LTO/MWCNTs is smaller than that of LTO nanosheets, indicating that the MWCNTs reduce the polarizations and speed up the electrochemical reaction 


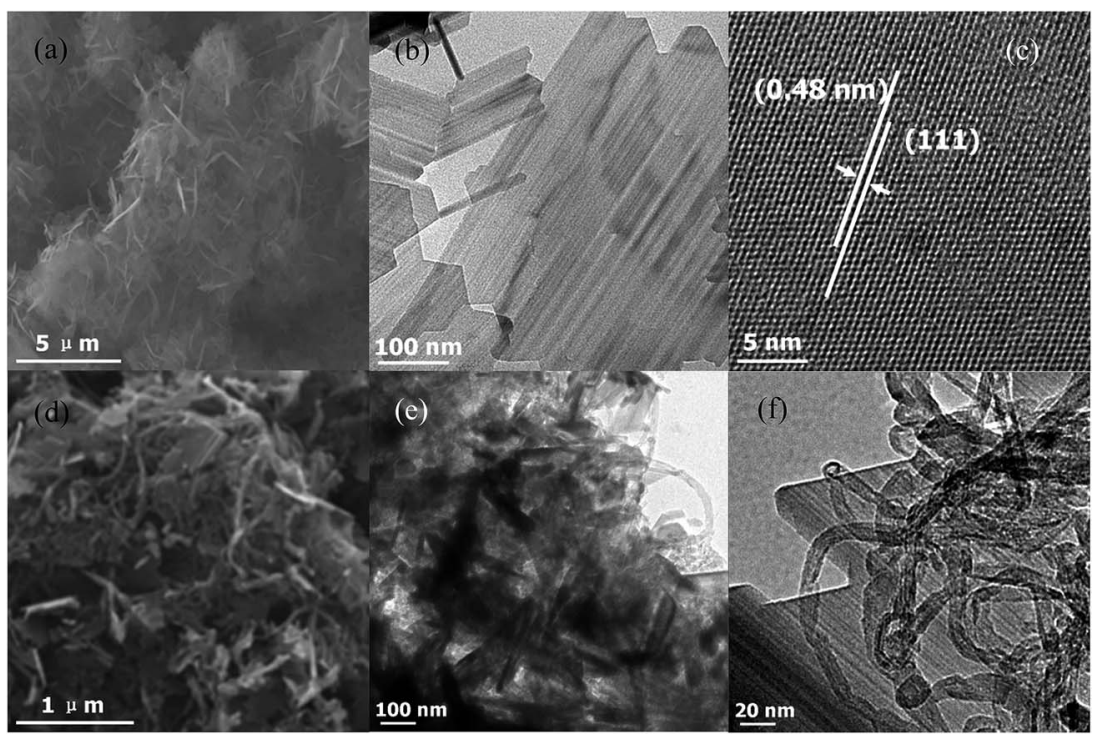

Fig. 3 (a-c) SEM, TEM and HRTEM images of crystalline LTO nanosheets; $(d-f)$ typical SEM and TEM images of the LTO/MWCNTs nanocomposite.

kinetics. Fig. 4b depicts the charge-discharge voltage profiles of the LTO/MWCNTs $(4: 1)$ electrodes for a few cycles at a current rate of $1 \mathrm{C}$ with a cut off window of 1.0-2.5 V. In agreement with the above $\mathrm{CV}$ analysis, two distinct voltage plateaus at around $1.55 \mathrm{~V}$ can be observed during the discharge and charge processes, which confirm that there is a two-phase reaction during electrochemical lithium insertion-extraction. Fig. 4c shows the rate capability of the LTO/MWCNTs $(4: 1)$ nanocomposite at various rates from 1 to $30 \mathrm{C}$. The specific capacity of the LTO/MWCNTs $(4: 1)$ electrode is superior to that of LTO and referenced W-LTO/WMCNTs composite at all charge/ discharge rates. For example, the capacities of the LTO/ MWCNTs $(4: 1)$ nanocomposite at the rate of $30 \mathrm{C}$ is 148.3 $\mathrm{mA} \mathrm{h} \mathrm{g}{ }^{-1}$. Nevertheless, the referenced LTO nanosheets deliver

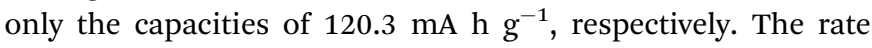
performances of three LTO/MWCNTs nanocomposites with different weight ratios of $8: 1,4: 1$ and $3: 2$ are also investigated from $1 \mathrm{C}$ to $30 \mathrm{C}$. The results shown in Fig. $4 \mathrm{~d}$ reveal that the LTO/MWCNTs $(4: 1)$ nanocomposite possess the best Li storage performance. To probe superior Li storage property of the LTO/MWCNTs nanocomposite, the cycling performance at a high current rate of $10 \mathrm{C}$ is investigated. Fig. 4e displays the cycling capacity of the LTO/MWCNTs $(4: 1)$ nanocomposite, demonstrating excellent capacity retention with only a loss of $10.2 \%$ after 500 cycles. The result is superior to that of the referenced W-LTO/MWCNTs composite and LTO nanosheets. The rate and cycling performance of the LTO/MWCNTs $(4: 1)$ nanocomposite are also comparable to the results found in the literatures according to Table SI. $\dagger$ In addition, the coulombic efficiency of the LTO/MWCNTs $(4: 1)$ nanocomposite approaches $100 \%$. The results imply rapid charge transport in the LTO/MWCNTs nanocomposite. To investigate enhanced transport kinetics, EIS measurements were conducted for the LTO/MWCNTs $(4: 1)$ nanocomposite as well as the W-LTO/
WMCNTs composite and LTO nanosheets after the rate capability test in Li half-cells. The typical Nyquist plots are shown in Fig. 4f, which are consisted of a common purely resistive response at high frequencies represented by ohmic resistance $\left(R_{\mathrm{S}}\right)$ of the electrode and electrolyte, a semicircle due to the charge-transfer impedance $\left(R_{\mathrm{ct}}\right)$ on the electrode-electrolyte interface in the high-to-middle frequency region, and an inclined straight line ascribed to the Warburg impedance $\left(Z_{\mathrm{w}}\right)$ in the low frequency region. The EIS spectra are simulated by the Z-view software using the inset equivalent circuit model and the corresponding simulation parameters are presented in Table 1. It can be observed that the LTO/MWCNTs $(4: 1)$ electrode has smaller charge-transfer resistance than those of the W-LTO/MWCNTs and LTO electrodes. Furthermore, the exchange current density $\left(i_{0}=R T / n F R_{\mathrm{ct}}\right)$ of the LTO/MWCNTs $(4: 1)$ electrode is higher than those of the referenced samples. The lithium ion diffusion coefficient $\left(D_{\mathrm{Li}}\right)$ can be evaluated by EIS according to the following equations: ${ }^{36,40}$

$$
\begin{array}{r}
D_{\mathrm{Li}}=\frac{R^{2} T^{2}}{2 A^{2} n^{4} F^{4} C^{2} \sigma^{2}} \\
Z^{\prime}=R_{\mathrm{s}}+R_{\mathrm{ct}}+\sigma \omega^{-1 / 2}
\end{array}
$$

where $R$ is the gas constant, $T$ is the absolute temperature, $A$ is the surface area of the electrode, $n$ is the number of electrons transferred in the half reaction of the redox couple, $F$ is Faraday's constant, $C$ is the concentration of lithium ions, and $\sigma$ is the Warburg factor. The value of $\sigma$ can be obtained from the slope of the lines between $Z^{\prime}$ and $\omega^{-1 / 2}$ as shown in Fig. S2a. $\dagger$ The calculated $D_{\mathrm{Li}}$ values of the electrodes presented in Table 1 reveal that the LTO/MWCNTs electrode has higher ion diffusion coefficient than the referenced ones. These results indicate that the unique integrated architecture of two-dimensional LTO nanosheets confined in MWCNTs networks not only improve 

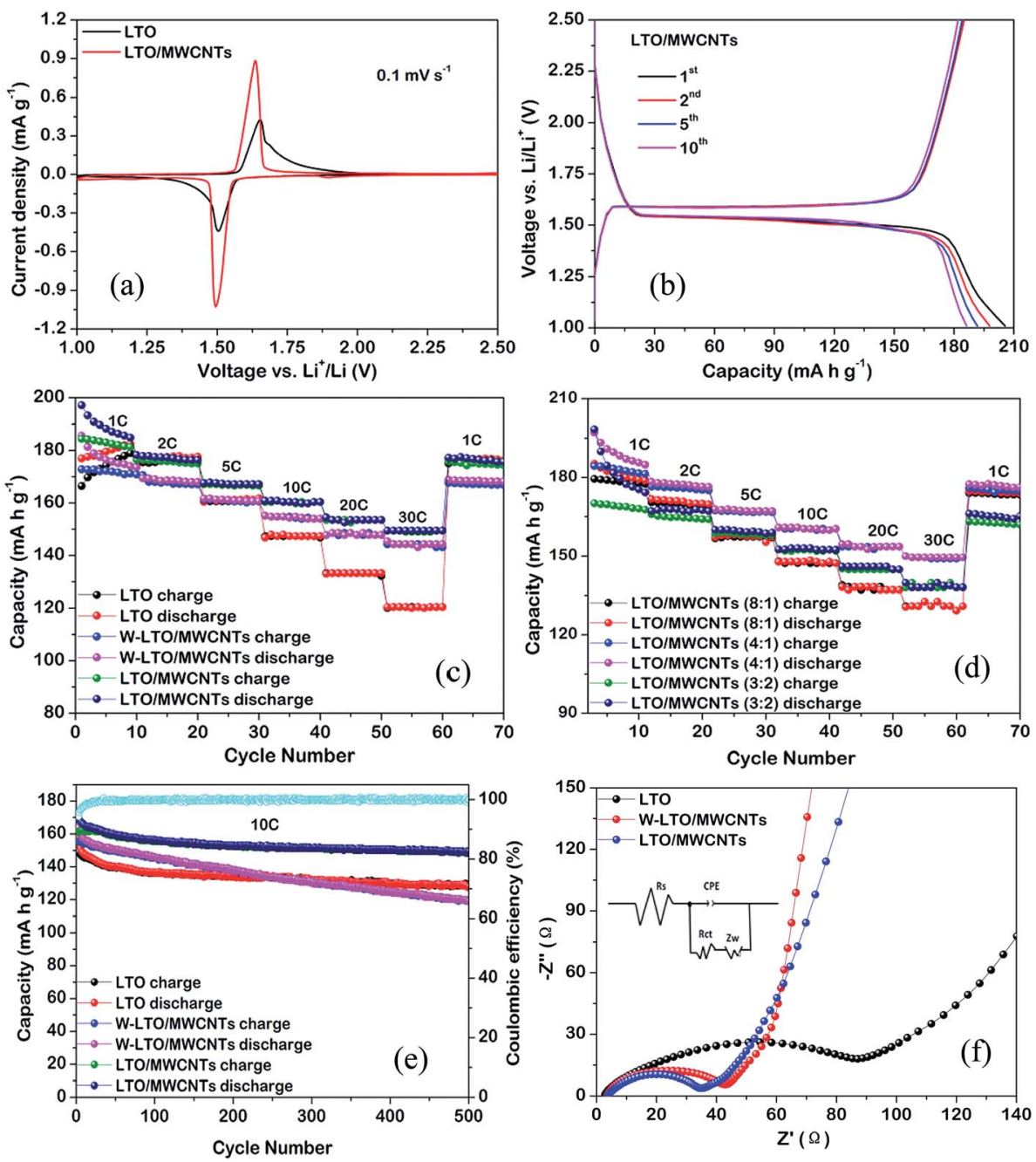

Fig. 4 (a) CV curves of LTO nanosheets and LTO/MWCNTs (4:1) nanocomposite at a scan rate of $0.1 \mathrm{mV} \mathrm{s}^{-1}$; (b) charge-discharge curves of LTO/MWCNTs $(4: 1)$ nanocomposite at $0.1 \mathrm{C}$ in Li half-cells; (c) rate performances of the LTO/MWCNTs $(4: 1)$ nanocomposite and reference samples; (d) rate performances of the LTO/MWCNTs nanocomposites with different weight ratios; (e) cycling performances of the LTO/ MWCNTs (4:1) nanocomposite and reference samples at rate of 10C; (f) Nyquist plots of the LTO/MWCNTs (4:1) nanocomposite and reference electrodes based on EIS after the rate capability test in Li half-cells. The inset shows the equivalent circuit used to fit the EIS.

Table 1 Calculated $R_{\mathrm{S}}, R_{\mathrm{ct}}, i_{0}$ and $D_{\mathrm{Li}}$ values for the LTO, W-LTO/ MWCNTS and LTO/MWCNTs electrodes in Li half-cells according to Fig. $4 f^{a}$

\begin{tabular}{lllll}
\hline Samples & $\begin{array}{l}R_{\mathrm{s}} \\
(\Omega)\end{array}$ & $\begin{array}{l}R_{\mathrm{ct}} \\
(\Omega)\end{array}$ & $\begin{array}{l}i_{0} \\
\left.(\mathrm{~mA} \mathrm{~cm})^{-2}\right)\end{array}$ & $\begin{array}{l}D_{\mathrm{Li}} \\
\left(\mathrm{cm}^{2} \mathrm{~s}^{-1}\right)\end{array}$ \\
\hline LTO & 5.73 & 88.1 & 0.291 & $3.8 \times 10^{-12}$ \\
W-LTO/MWCNTs $(4: 1)$ & 4.63 & 40.7 & 0.630 & $5.1 \times 10^{-12}$ \\
LTO/MWCNTs $(4: 1)$ & 4.24 & 30.6 & 0.838 & $1.01 \times 10^{-11}$
\end{tabular}

${ }^{a} i_{0}=R T / n F R_{\mathrm{ct}}$.

charge conductivity, but also decrease the polarization of LTO/ MWCNTs electrodes, thereby enhancing rate capability and cycling performance.

Since LTO anode materials is also a good materials for SIBs, sodium ion insertion-extraction behavior of the LTO/MWCNTs $(4: 1)$ nanocomposite is tested. With respect to the application of LTO to SIBs, $\mathrm{Na}^{+}$insertion and extraction are realized via a three-phase separation mechanism according to following equation: ${ }^{19} 2 \mathrm{Li}_{4} \mathrm{Ti}_{5} \mathrm{O}_{12}+6 \mathrm{Na}^{+}+6 \mathrm{e}^{-} \rightarrow \mathrm{Li}_{7} \mathrm{Ti}_{5} \mathrm{O}_{12}+\mathrm{LiNa}_{6} \mathrm{Ti}_{5} \mathrm{O}_{12}$. The incompletely reverse three-phase separation reaction produces a new phase of $\mathrm{LiNa}_{6} \mathrm{Ti}_{5} \mathrm{O}_{12}$ besides the rocksalt $\mathrm{Li}_{7} \mathrm{Ti}_{5} \mathrm{O}_{12}$ and induces a volume change of $\sim 13 \%$. In addition, larger ionic radius of $\mathrm{Na}^{+}(1.02 \AA)$ than that of $\mathrm{Li}^{+}(0.76 \AA)$ and slower $\mathrm{Na}^{+}$diffusion coefficient compared to the $\mathrm{Li}^{+}$diffusion coefficient in LTO raises the requirement for the electrode materials for SIBs. As a result, inferior electrochemical performances of the prepared LTO-based materials for SIBs relative to LIBs are often observed. Firstly, electrochemical CV analyses of the LTO/MWCNTs $(4: 1)$ nanocomposite and LTO nanosheets were characterized from 0.5 to $2.5 \mathrm{~V} v s$. $\mathrm{Na}^{+} / \mathrm{Na}$ at a scanning rate of $0.1 \mathrm{mV} \mathrm{s}^{-1}$. As depicted in Fig. $5 \mathrm{~b}$, a pair of oxidation and reduction peaks at $\sim 1.09$ and $\sim 0.67 \mathrm{~V}$, representing typical $\mathrm{Na}^{+}$ insertion/extraction in the LTO crystal lattice, can be observed for LTO nanosheets electrode. Besides, there exist a tiny 

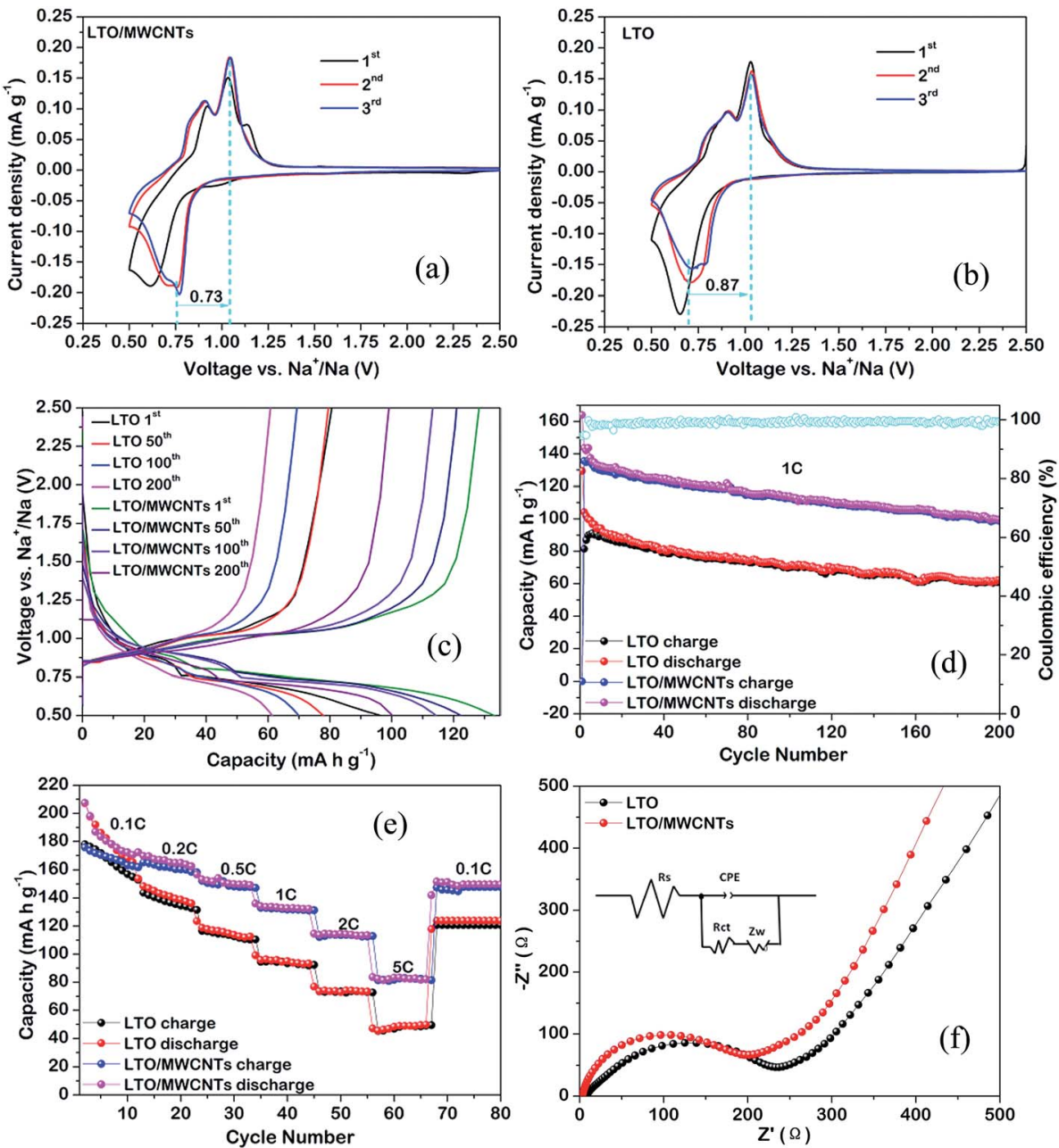

Fig. 5 ( $a$ and b) CV curves of the LTO/MWCNTs (4:1) and LTO electrodes at a scan rate of $0.1 \mathrm{mV} \mathrm{s}^{-1}$ in Na half-cells; (c) the charge-discharge curves of at different cycles in Na half-cells; ( $d$ and e) corresponding cycling performance at rate of $2 \mathrm{C}$ and rate performance of the LTO/ MWCNTs $(4: 1)$ and LTO electrodes; ( $f$ ) Nyquist plots of LTO/MWCNTs $(4: 1)$ and reference electrodes based on EIS after the rate capability test in $\mathrm{Na}$ half-cells. The inset shows the equivalent circuit used to fit the EIS.

oxidation peak at around $0.9 \mathrm{~V}$, implying that $\mathrm{Na}^{+}$extraction process is a more complicated pathway. ${ }^{41}$ These features are consistent with those from the LTO/MWCNTs electrode (see Fig. 5a). More importantly, the voltage difference between oxidation and reduction peaks of the LTO/MWCNTs electrode $(0.73 \mathrm{~V})$ is smaller than that of the LTO nanosheets electrode $(0.87 \mathrm{~V})$. These results indicate that the LTO/MWCNTs electrode as an anode for SIBs has improved electronic conductivity and depressed electrochemical polarization. Fig. $5 c$ depicts the charging-discharging profiles of the LTO NSs and LTO/ MWCNTs electrodes for the $1^{\text {st }}, 100^{\text {th }}$ and $200^{\text {th }}$ cycles at a current rate of $2 \mathrm{C}$ within the voltage range of $0.5-2.5 \mathrm{~V} v s . \mathrm{Na}^{+} /$ $\mathrm{Na}$. The samples present similar flat voltage plateaus at around $0.66 \mathrm{~V}$ and $1.09 \mathrm{~V}$, which is consistent with the CV results. Fig. $5 \mathrm{~d}$ shows the rate capability of the LTO/MWCNTs $(4: 1)$ nanocomposite as an anode for SIBs at various rates. The LTO/ MWCNTs (4:1) nanocomposite exhibits reversible capacities of 161.5, 147.1, 131.7, and 113.3 at rates of $0.2,0.5,1$, and 2C, respectively. Even at a high rate of $5 \mathrm{C}$, a reversible capacity of $82.7 \mathrm{~mA} \mathrm{~h} \mathrm{~g}^{-1}$ is retained. When the current rate is reset to $0.1 \mathrm{C}$ after cycling at various rates, the capacity can recover to 147.2 $\mathrm{mA} \mathrm{h} \mathrm{g}^{-1}$. With regard to the cycling durability, the cycling performance is investigated at a large current density of 1C. As shown in Fig. 5e, stable reversible capacity of the LTO/MWCNTs (4:1) nanocomposite can be retained to $99 \mathrm{~mA} \mathrm{~h} \mathrm{~g}^{-1}$ after 200 cycles. The rate performance and cycling durability of the LTO/ MWCNTs $(4: 1)$ nanocomposite are superior to those of the referenced LTO nanosheets and comparable to the reported results (see Table SII $\dagger$ ). These results demonstrate that the LTO/ MWCNTs anode is suitable for high power SIBs. Furthermore,

Table 2 Calculated $R_{\mathrm{s}}, R_{\mathrm{ct}}, i_{\mathrm{O}}$ and $D_{\mathrm{Na}}$ values for the LTO and LTO/ MWCNTs electrodes in Na half-cells according to Fig. $5 f^{a}$

\begin{tabular}{lllll}
\hline Samples & $\begin{array}{l}R_{\mathrm{s}} \\
(\Omega)\end{array}$ & $\begin{array}{l}R_{\mathrm{ct}} \\
(\Omega)\end{array}$ & $\begin{array}{l}i_{0} \\
\left(\mathrm{~mA} \mathrm{~cm}^{-2}\right)\end{array}$ & $\begin{array}{l}D_{\mathrm{Na}} \\
\left(\mathrm{cm}^{2} \mathrm{~s}^{-1}\right)\end{array}$ \\
\hline LTO & 5.03 & 205 & 0.125 & $2.1 \times 10^{-14}$ \\
LTO/MWCNTs $(4: 1)$ & 4.66 & 181 & 0.142 & $4.6 \times 10^{-14}$
\end{tabular}

${ }^{a} i_{0}=R T / n F R_{\mathrm{ct}}$. 
EIS measurements (see Fig. 5f) with the simulated results (see Table 2) reveal that the LTO/MWCNTs electrode has lower charge-transfer resistance, higher exchange current density and larger sodium ion diffusion coefficient $\left(D_{\mathrm{Na}}\right)$ compared to the referenced electrode. The results further confirm that the unique integrated architecture of two-dimensional LTO nanosheets confined in MWCNTs networks benefit the improvement of transport kinetics in the nanocomposite electrode.

\section{Conclusions}

In summary, we have fabricated a nanocomposite composed of crystalline LTO nanosheets and MWCNTs via a facile heteroassembly process and followed thermal treatment. During fabrication, as electrostatic repulsion among MWCNTs with negative charges is effectively screened, the MWCNTs assemble well with the introduced LTO nanosheets with abundant charged ions, thereby producing robust inner-connected architecture of 2D LTO nanosheets embedded in highly porous and electrically conductive MWCNTs network, which is very beneficial to promote electrochemical reaction kinetics. As anodes for LIBs and SIBs, the LTO/MWCNTs nanocomposite demonstrates outstanding lithium and sodium storage performances. As for lithium storage, the anode delivers reversible capacity of $149.7 \mathrm{~mA} \mathrm{~h} \mathrm{~g}^{-1}$ at 10C after 500 cycles. In terms of sodium storage, it has reversible capacity of $82 \mathrm{~mA} \mathrm{~h} \mathrm{~g}^{-1}$ at $5 \mathrm{C}$ and high stable capacity of $99 \mathrm{~mA} \mathrm{~h} \mathrm{~g}^{-1}$ at $1 \mathrm{C}$ after 200 cycles. The results suggest that the developed LTO/MWCNTs nanocomposite is a promising anode material for high-performance LIBs and SIBs.

\section{Acknowledgements}

This work was financially supported by the Grants from National Natural Science Foundation of China (No. 11474242 and 51272220), Program for Changjiang Scholars and Innovative Research Team in University (Grants No. IRT13093) and the Hunan Provincial Innovation Foundation for Graduate (No. CX2016B254).

\section{References}

1 J. Liu, N. Li, M. D. Goodman, H. G. Zhang, E. S. Epstein, B. Huang, Z. Pan, J. Kim, J. H. Choi and X. Huang, ACS Nano, 2015, 9, 1985-1994.

2 I. Hasa, D. Buchholz, S. Passerini and J. Hassoun, ACS Appl. Mater. Interfaces, 2015, 7, 5206-5212.

3 I. Hasa, R. Verrelli and J. Hassoun, Electrochim. Acta, 2015, 173, 613-618.

4 L. David, R. Bhandavat and G. Singh, ACS Nano, 2014, 8, 1759-1770.

5 E. Yang, H. Ji and Y. Jung, J. Phys. Chem. C, 2015, 119, 2637426380.

6 C. Wu, P. Kopold, Y.-L. Ding, P. A. v. Aken, J. Maier and Y. Yu, ACS Nano, 2015, 9, 6610-6618.
7 D. Wang, N. Chen, M. Li, C. Wang, H. Ehrenberg, X. Bie, Y. Wei, G. Chen and F. Du, J. Mater. Chem. A, 2015, 3, 8636-8642.

8 G. Pang, C. Yuan, P. Nie, B. Ding, J. Zhu and X. Zhang, Nanoscale, 2014, 6, 6328-6334.

9 Y. Ma, B. Ding, G. Ji and J. Y. Lee, ACS Nano, 2013, 7, 1087010878.

10 J. Liu, K. Song, P. A. v. Aken, J. Maier and Y. Yu, Nano Lett., 2014, 14, 2597-2603.

11 C. Kim, N. S. Norberg, C. T. Alexander, R. Kostecki and J. Cabana, Adv. Funct. Mater., 2013, 23, 1214-1222.

12 L. Shen, E. Uchaker, X. Zhang and G. Cao, Adv. Mater., 2012, 24, 6502-6506.

13 H. Li, L. Shen, J. Wang, B. Ding, P. Nie, G. Xu, X. Wang and X. Zhang, ChemPlusChem, 2014, 79, 128-133.

14 C. Han, Y. B. He, B. Li, H. Li, J. Ma, H. Du, X. Qin, Q. H. Yang and F. Kang, ChemSusChem, 2014, 7, 2567-2574.

15 G. Xu, L. Yang, X. Wei, J. Ding, J. Zhong and P. K. Chu, Adv. Funct. Mater., 2016, 26, 3349-3358.

16 C. Chen, H. Xu, T. Zhou, Z. Guo, L. Chen, M. Yan, L. Mai, P. Hu, S. Cheng and Y. Huang, Adv. Energy Mater., 2016, 6, 201600322.

17 P. Yu, C. Li and X. Guo, J. Phys. Chem. C, 2014, 118, 1061610624.

18 X. Yu, H. Pan, W. Wan, C. Ma, J. Bai, Q. Meng, S. N. Ehrlich, Y.-S. Hu and X.-Q. Yang, Nano Lett., 2013, 13, 4721-4727.

19 Y. Sun, L. Zhao, H. Pan, X. Lu, L. Gu, Y.-S. Hu, H. Li, M. Armand, Y. Ikuhara, L. Chen and X. Huang, Nat. Commun., 2013, 4, 1870.

20 Z. Liang, P. Hui-Lin, H. Yong-Sheng, L. Hong and C. Li-Quan, Chin. Phys. B, 2012, 21, 028201.

21 Q. Zhou, L. Liu, J. Tan, Z. Yan, Z. Huang and X. Wang, J. Power Sources, 2015, 283, 243-250.

22 K.-T. Kim, C.-Y. Yu, C. S. Yoon, S.-J. Kim, Y.-K. Sun and S.-T. Myung, Nano Energy, 2015, 12, 725-734.

23 L. Shen, X. Zhang, E. Uchaker, C. Yuan and G. Cao, Adv. Energy Mater., 2012, 2, 691-698.

24 M. R. Jo, K. M. Nam, Y. Lee, K. Song, J. T. Park and Y.-M. Kang, Chem. Commun., 2011, 47, 11474-11476.

25 J. Chen, L. Yang, S. Fang and Y. Tang, Electrochim. Acta, 2010, 55, 6596-6600.

26 N. Li, G. Zhou, F. Li, L. Wen and H. M. Cheng, Adv. Funct. Mater., 2013, 23, 5429-5435.

27 L. Y. Y. Tang, S. Fang and Z. Qiu, Electrochim. Acta, 2009, 54, 6244-6249.

28 L. Yu, H. B. Wu and X. W. D. Lou, Adv. Mater., 2013, 25, 22962300.

29 J. Cheng, R. Che, C. Liang, J. Liu, M. Wang and J. Xu, Nano Res., 2014, 7, 1043-1053.

30 L. Shen, C. Yuan, H. Luo, X. Zhang, K. Xu and F. Zhang, J. Mater. Chem., 2011, 21, 761-767.

31 G. Du, B. R. Winton, I. M. Hashim, N. Sharma, K. Konstantinov, M. Reddy and Z. Guo, RSC Adv., 2014, 4, 38568-38574.

32 Y.-Q. Wang, L. Gu, Y.-G. Guo, H. Li, X.-Q. He, S. Tsukimoto, Y. Ikuhara and L.-J. Wan, J. Am. Chem. Soc., 2012, 134, 78747879 . 
33 L. Gao, S. Li, D. Huang, Y. Shen and M. Wang, J. Mater. Chem. A, 2015, 3, 10107-10113.

34 L. Gao, S. Li, D. Huang, Y. Shen and M. Wang, J. Mater. Chem. A, 2015, 3, 23570-23576.

35 S. Chen, Y. Xin, Y. Zhou, Y. Ma, H. Zhou and L. Qi, Energy Environ. Sci., 2014, 7, 1924-1930.

36 G. Xu, L. Yang, X. Wei, J. Ding, J. Zhong and P. Chu, J. Power Sources, 2015, 295, 305-313.

37 Z. Wu, G. Xu, X. Wei and L. Yang, Electrochim. Acta, 2016, 207, 275-283.
38 J. Ming, Y. Wu, Y. Yu and F. Zhao, Chem. Commun., 2011, 47, 5223-5225.

39 G. B. Xu, L. W. Yang, X. L. Wei, J. W. Ding, J. X. Zhong and P. K. Chu, J. Power Sources, 2016, 327, 580-590.

40 G. B. Xu, W. Li, L. W. Yang, X. L. Wei, J. W. Ding, J. X. Zhong and P. K. Chu, J. Power Sources, 2015, 276, 247-254.

41 G. Hasegawa, K. Kanamori, T. Kiyomura, H. Kurata, K. Nakanishi and T. Abe, Adv. Energy Mater., 2015, 5, 1400730. 